Pacific Journal of Mathematic 


\title{
DENSE STRONG CONTINUITY OF POINTWISE CONTINUOUS MAPPINGS
}

\author{
Petar Kenderov
}

Let $Y$ be a topological space and $Z$ be a metric space with metric $d(\cdot, \cdot)$. Denote by $C(Y, Z)$ the space of all continuous functions from $Y$ into $Z$. For a given topological space $X$ and a pointwise continuous mapping $T: X \rightarrow C(Y, Z)$ a theorem is proved asserting (under some conditions) that $T$ is continuous at the points of some dense $G_{\delta}$ subset of $X$ with respect to the topology of uniform convergence in $C(Y, Z)$. A "set-valued" version of this result is also proved. It is shown how one can use these results in order to get new information about points of continuity and singlevaluedness of (multivalued) monotone operators and (multivalued) metric projections. As corollaries some known results about Gâteaux or Fréchet differentiability of convex functions on a dense subset of their domains of continuity are obtained.

o. Introduction. Let $Y$ be a compact topological space and $Z$ be a metric space. By $C(Y, Z)$ we denote, as usual, the space of all continuous functions from $Y$ into $Z$. In $C(Y, Z)$ we will consider two topologies. The topology of pointwise convergence and the topology of uniform convergence on $Y$. Throughout this introduction $X$ will always denote either a complete metric space or a compact topological space.

Let $T: X \rightarrow C(Y, Z)$ be a mapping which is continuous with respect to the pointwise topology in $C(Y, Z)$. I. Namioka (Pacific J. Math., 51 (1974), 515-531, Theorem 2.2) proved that there is a dense $G_{i}$-subset $A$ of $X$ such that, at each point of $A$, the mapping $T$ is continuous relative to the topology of uniform convergence in $C(Y, Z)$.

If $E$ is a real normed space and $B^{*}$ is the unit ball of the dual space with the weak* topology, then $E$ can be considered as a subset of $C\left(B^{*}, R\right)$, where $R$ is the real line. The pointwise convergence topology in $C\left(B^{*}, R\right)$ coincides in $E \subset C\left(B^{*}, R\right)$ with the usual weak topology of the normed space $E$ and the topology of uniform convergence in $C\left(B^{*}, R\right)$ induces the norm topology in $E$. This is why the above mentioned result of Namioka implies the following one: Let $T: X \rightarrow E$ be a continuous mapping from the (compact or complete metric) space $X$ into $E$ with its weak topology. Then there is a dense $G_{\delta}$-subset $A$ of $X$ such that, at each point of $A$, the mapping $T$ is continuous relative to the norm topology in $E$. 
As an immediate corollary Namioka obtained from this result that, if $X$ is a weak compact subset of the normed space $E$, then the identity map $(X$, weak $) \rightarrow(X$, norm $)$ is continuous at the points of some dense $G_{\dot{o}}$-subset of ( $X$, weak). This result contains a lot of information. For instance, by means of it Namioka proved that every weakly compact convex subset of $E$ is the closed convex hull of its denting points. However, the question of norm continuity at some points of a given weak ${ }^{*}$-continuous mapping $T: X \rightarrow\left(E^{*}\right.$, weak*), where $E^{*}$ is the dual of $E$, is more complicated. There exists a Banach space $E$ and a weak*-compact subset $K$ of $E^{*}$ such that the identity mapping $\left(K\right.$, weak $\left.{ }^{*}\right) \rightarrow\left(E^{*}\right.$, norm $)$ is not continuous at any point of $K$. Nevertheless Namioka gave in the same paper two positive results. If $E^{*}$ has one of the two properties mentioned below, then the identity mapping $\left(K\right.$, weak $\left.{ }^{*}\right) \rightarrow\left(E^{*}\right.$, norm $)$, where $K$ is a weak ${ }^{*}$-compact subset of $E^{*}$, is continuous at the points of some dense $G_{j}$-subset of $\left(K\right.$, weak $\left.{ }^{*}\right)$. These two properties are:

(a) $\left(E^{*}\right.$, norm $)$ is weak-compactly generated (i.e., there is a weakly compact set $C$ of ( $E^{*}$, norm) such that the linear span of $C$ is dense in $\left(E^{*}\right.$, norm)).

(b) There is an equivalent norm in $E$ such that its dual norm $\|\cdot\|^{*}$ satisfies the condition: for any net $\left\{f_{\alpha}\right\}_{\alpha} \subset E^{*}$, it follows from $\left\|f_{\alpha}\right\|^{*} \rightarrow\|f\|^{*}$ and weak ${ }^{*} \lim _{\alpha} f_{\alpha}=f$ that $\lim _{\alpha}\left\|f_{\alpha}-f\right\|^{*}=0$.

In this connection it is natural to look for all those Banach spaces $E$ for which any weak*-continuous mapping $T: X \rightarrow\left(E^{*}\right.$, weak $^{*}$ ) is norm continuous at the points of some dense $G_{i}$-subset of $X$. It turns out this is the case if and only if every separable subspace of $E$ has a separable dual (equivalently, if and only if $E^{*}$ has the Radon-Nikodym property or, if and only if every continuous convex real-valued function in $E$ is Frechet differentiable at the points of some dense $G_{\dot{\delta}}$-subset of its domain of continuity (in the latter case the space $E$ is called "Asplund space")).

This result as well as the above mentioned result of Namioka are obtained in this paper as corollaries of one and the same general theorem. The proof of this theorem is, in essence, identical with the classical Cantor-set-construction. The Cantor-set-construction was already used for proving similar kind of theorems. C. Stegall (Trans. Amer. Math. Soc., 206 (1975), 213-223) proved that, if $E^{*}$ has the Radon-Nikodym property, then every separable subspace of $E$ has a separable dual. After that Namioka, basing on a proof of Stegall, adapted the Cantor-set-construction to give a proof of the fact that, if every separable subspace of $E$ has separable dual,then every weak*-compact subset of $E^{*}$ contains relatively weak*-open subsets of arbitrarily small diameter. It was this proof of Namioka (J. Diestel, J. J. Uhl, Jr. "Vector Measures", Amer. Math. Soc. Math. 
Surveys No. 15, p. 213) that suggested to the author the idea how to use Cantor-set-construction in proving the results contained in this paper. We also prove a general "multivalued version" of the above continuity result. As a partial case we have: If $T: X \rightarrow$ $(E$, weak) is an upper semicontinuous multivalued mapping with convex and weak-compact images: then at every point $x$ from some dense $G_{\dot{\delta}}$-subset $A$ of $X$ the mapping $T$ has the following "continuity property"

(cp) such that $\inf \left\{\left\|f^{\prime}-f^{\prime \prime}\right\|: f^{\prime} \in T x^{\prime}, f^{\prime \prime} \in T x^{\prime \prime}\right\} \leqq \varepsilon$ whatevery $x^{\prime}, x^{\prime \prime} \in U$.

In some particular situations, when the mapping $T$, the space $X$ and the space $E$ are chosen in an appropriate way, the points at which $T$ satisfies the (cp)-condition turn out to be exactly the continuous linear functionals $\in E^{*}$ which strongly expose a given weakly compact subset of $E$. Or, more generally, the points where $T$ satisfies $(c p)$ are the points at which a given convex function is Frechet differentiable. In this way we give a direct proof, without introducing the notion of "dentability" or the Radon-Nikodym property, of the fact that, if every separable subspace of $E$ has a separable dual, then each continuous covex real-valued function defined in $E$ is Frechet differentiable at the points of some dense $G_{\dot{o}}$-subset of $E$.

In another situation (under another choice of $T, X$ and $E$ ), the points with $(c p)$ are exactly those points where a given (multivalued metric projection is single-valued and norm-to-norm upper semicontinuous. The same $(c p)$-condition allows us to obtain new information about continuity property of (multivalued) monotone operators defined in weak-compactly generated Banach spaces.

1. Strongly countably complete spaces and the Cantor-setconstruction for single-valued mappings. Let $\gamma$ be an open covering of a topological space $X$. Some subset $S$ of $X$ is called $\gamma$-small if $S$ is contained in a member of $\gamma$. The topological space $X$ is said to be strongly countably complete (Frolik [8]) if there exists a sequence $\left\{\gamma_{i}: i=1,2, \cdots\right\}$ of open coverings of $X$ such that a decreasing sequence $\left\{F_{i}\right\}_{i \geqq 1}$ of closed subsets of $X$ has nonempty intersection whenever each $F_{i}$ is $\gamma_{i}$-small and nonempty. We will consider only Hausdorff and regular strongly countably complete spaces $X$ in this paper. This class of spaces is very large. Every locally countably compact space is strongly countably complete. In particular every (locally) compact topological space is strongly countably complete. Every complete metric space is also countably complete 
(as the covering $\gamma_{n}, n=1,2,3$, one takes the collection of open balls with radius $1 / n)$. The same argument as in the classical Baire theorem shows that the intersection of every sequence of dense open subsets of the strongly countably complete space $X$ is again a dense subset of $X$.

By $C(Y, Z)$, where $Y$ and $Z$ are topological spaces, we denote the set of all continuous maps $f: Y \rightarrow Z$. For an arbitrary subset $Y_{1}$ of $Y$ we denote by $r_{Y_{1}}: C(Y, Z) \rightarrow C\left(Y_{1}, Z\right)$ the "restriction map" assigning to each $f$ from $C(Y, Z)$ its restriction on $Y_{1}$. In $C(Y, Z)$ we will consider different topologies. The simplest one is the pointwise convergence topology which will be denoted by " $p$ ". When $Z$ is a metrizable space, we consider in $C(Y, Z)$ something like the uniform convergence topology. Suppose $d(\cdot, \cdot)$ is a metric in $Z$ generating its topology. For a given $f_{0}$ from $C(Y, Z)$ and a positive number $t$ put $U\left(f_{0}, t\right)=\left\{f \in C(Y, Z): d\left(f, f_{0}\right):=\sup \left\{d\left(f(y), f_{0}(y)\right): y \epsilon\right.\right.$ $Y\}<t\}$. The sets $\{U(f, t): f \in C(Y, Z), t>0\}$ form a base for some topology in $C(Y, Z)$. This topology will be denoted by " $u$ ". It depends on the particular choice of the metric $d(\cdot, \cdot)$ and may change if in the definition of " $u$ " $d(\cdot, \cdot)$ is replaced by another equivalent metric in $Z$. However, when $Y$ is a compact space, all equivalent metrics in $Z$ generate one and the same topology in $C(Y, Z)$ and this is just the uniform convergence topology. This is why the above defined topology " $u$ " in $C(Y, Z)$ will be called in the sequel (even in the case of noncompact $Y$ ) "uniform convergence topology". Correspondingly, the function $d\left(f_{1}, f_{2}\right)=\sup \left\{d\left(f_{1}(y), f_{2}(y)\right): y \in Y\right\}$, where $f_{1}, f_{2} \in C(Y, Z)$, will be referred to as "uniform metric in $C(Y, Z)$ " even though $d\left(f_{1}, f_{2}\right)$ might be equal to plus infinity for some $f_{1}, f_{2} \in C(Y, Z)$. For a given mapping $T: X \rightarrow C(Y, Z)$ we use the following expressions as synonyms: " $T$ is continuous at $x_{0} \in X$ with respect to $p$ ", " $T$ is $p$-continuous at $x_{0} \in X$ " and " $T: X \rightarrow$ $(C(Y, Z), p)$ is continuous at $x_{0} \in X$ ". This agreement is also valid for every other topology (instead of $p$ ) which appears in our text.

We are now ready to formulate a general sufficient condition for a given continuous mapping $T: X \rightarrow(C(Y, Z), p)$ to be $u$-continuous at the points of some "fat" subset of $X$.

THEOREM 1.1. Let $X$ be a strongly countably complete space, $Y$ a topological space, $Z$ a metric space and let $T: X \rightarrow(C(Y, Z), p)$ be a continuous mapping such that, for every countably compact set $\Delta \subset X$ and every closed separable $Y_{1} \subset Y$, the set $r_{Y_{1}} \circ T \Delta$ is a separable subset of $\left(C\left(Y_{1}, Z\right), u\right)$. Then there exists a dense $G_{\dot{o}}$-subset $A$ of $X$ such that $T: X \rightarrow(C(Y, Z), u)$ is continuous at every $x \in A$.

Before giving the proof of this theorem we want to show two 
important for our applications examples in which the requirement of the theorem is fulfilled for every $p$-continuous mapping $T: X \rightarrow$ $C(Y, Z)$.

Proposition 1.2. Let $Y$ be a compact space and $Z$ be a metric space. Suppose further $T: X \rightarrow C(Y, Z)$ is a p-continuous map from the topological space $X$ into $C(Y, Z)$. Then, for every countably compact subset $\triangle$ of $X$ and every closed separable subset $Y_{1}$ of $Y$, the set $r_{Y_{1}} \circ T \Delta$ is a separable subset of $\left(C\left(Y_{1}, Z\right), u\right)$.

Proof of Proposition 1.2. Put $r:=r_{Y_{1}}$ and $A=r \circ T \Delta . r \circ T: X \rightarrow$ $\left(C\left(Y_{1}, Z\right), p\right)$ is continuous and therefore the set $A$ is also countably compact. By a result of Troallic ([18], Proposition 1) $A$ is $u$-separable if and only if it is $p$-separable. Hence it is enough to prove that $A$ is a separable subset of $\left(C\left(Y_{1}, Z\right), p\right)$. To prove this we remark first that in $C\left(Y_{1}, Z\right)$ there is a metrizable topology " $m$ " which is weaker than $p$. This topology " $m$ " is just the topology of pointwise convergence at the points of some countable and dense subset of $Y_{1}$. This means that the set $A$ is $m$-countably compact and consequently $m$-compact. As any other metrizable compact, the space $(A, m)$ has a countable dense subset $C \subset A$. We prove now that $C$ is dense in $(A, p)$ as well. Indeed, take $f_{0} \in A$. Since $C$ is dense in $(A, m)$ we find a sequence $\left(f_{i}\right)_{i \geqq 1} \subset C m$-converging to $f_{0}$. As $(A, p)$ is countably compact, the sequence $\left(f_{i}\right)_{i \geqq 1}$ must have a $p$-cluster point $\bar{f} \in A$. But the topology $p$ is stronger than $m$ and $\bar{f}$ is also $m$-cluster point of the sequence $\left(f_{i}\right)_{i \geqq 1}$. Since the latter sequence has only one $m$-cluster point and this is $f_{0}$, we get $\bar{f}=f_{0}$. Thus $(A, p)$ is separable and Proposition 1.2 is proved.

Let now $E$ be a real Banach space and $E^{*}$ be its dual. Denote the unit ball of $E$ by $B:=\{x \in E:\|x\| \leqq 1\}$. The set $E^{*}$ can be considered as a subset of $C(B, R)$, where $R$ is the usual real line. It is clear that the usual pointwise convergence topology $p$ in $C(B, R)$ induces the weak ${ }^{*}$ topology of $E^{*}$ and the uniform convergence topology $u$ of $C(B, R)$ generates the norm topology of $E^{*}$. By " $w^{*}$ " and " $n^{*}$ " we will denote the weak" and the norm topology of $E^{*}$ correspondingly. " $w$ " and " $n$ " are reserved for the weak and the norm topology of $E$. When there is no danger of ambiguity we will omit the star in " $n$ "”.

Let $Y_{1}$ be a closed separable subset of $(B, n)$. The restriction mapping $r: C(B, R) \rightarrow C\left(Y_{1}, R\right)$ turning every $f$ from $C(B, R)$ into its restriction on $Y_{1}$ will assign to every $f^{*} \in E^{*}$ its restriction on $Y_{1}$ and the latter restriction can be identified (in a one-to-one way) with a continuous linear functional defined on the closed linear span $L$ of $Y_{1}$ in $E$. Thus $r\left(E^{*}\right)$ is a subset of $L^{*}$. Moreover, the 
$n^{*}$-topology of $L^{*}$ is stronger than (or equal to) the one inherited from $\left(C\left(Y_{1}, R\right), u\right)$. One needs no more argument for the following.

Proposition 1.3. Suppose that every closed separable linear subspace of the Banach space $E$ has a separable dual. Consider $E^{*}$ as a subset of $C(B, R)$. Then the image of $E^{*}$ under any "restriction mapping" defined by a closed separable subset $Y_{1}$ of $(B, n)$ is a separable subset of $\left(C\left(Y_{1}, R\right), u\right)$ (i.e., the requirement of Theorem 1.1 is fulfilled for every weak*-continuous mapping $T: X \rightarrow E^{*} \subset$ $C(B, R))$.

We turn now back to the proof of Theorem 1.1. Let $T: X \rightarrow$ $C(Y, Z)$ be as in the formulation of the theorem and let $n$ be a positive integer. Put $H_{n}=\{x \in X$ : for every open $U, x \in U$, the diameter of the set $T U \subset C(Y, Z)$ with respect to the uniform metric is greater than $1 / n\}$. Clearly, the condition $x_{0} \notin \bigcup_{n \geq 1} H_{n}$ implies that $T: X \rightarrow(C(Y, Z), u)$ is continuous at $x_{0}$. Thus, what we have to prove is that $H_{n}$ is nowhere dense in $X$ (the set $H_{n}$ is also closed in $X$ but we are not interested in this at the moment). We will make a repeated use of the following observation.

LEMMA 1.4. Let $\gamma$ be an open covering of $X$ and $U$ be an open subset of $X$ with $U \cap H_{n} \neq \varnothing$. Then there exist two nonempty open sets $U^{\prime}, U^{\prime \prime} \subset U$ and a point $y \in Y$ such that:

(i) the closures $\bar{U}^{\prime}, \bar{U}^{\prime \prime}$ in $X$ of the sets $U^{\prime}, U^{\prime \prime}$ are contained in $U$ and are $\gamma$-small sets.

(ii) for every $x^{\prime} \in U^{\prime}$ and every $x^{\prime \prime} \in U^{\prime \prime}$, the inequality $d\left(T x^{\prime}(y), T x^{\prime \prime}(y)\right)>1 / n$ holds (where $d(\cdot, \cdot)$ is the metric in $Z$ ).

Proof of Lemma 1.4. Take some open $U \subset X$ with $U \cap H_{n} \neq \varnothing$. By the definition of $H_{n}$, diam $T U>1 / n$. This means that we can find $x_{c}^{\prime}, x_{0}^{\prime \prime} \in U$ and an $y \in Y$ such that $d\left(T x_{0}^{\prime}(y), T x_{0}^{\prime \prime}(y)\right)>1 / n$. Put $\beta=\left(d\left(T x_{0}^{\prime}(y), T x_{0}^{\prime \prime}(y)\right)-(1 / n)\right) / 2$ and consider the sets $V^{\prime}=\{f \in$ $\left.C(Y, Z): d\left(f(y), T x_{0}^{\prime}(y)\right)<\beta\right\}$ and $V^{\prime \prime}=\left\{f \in C(Y, Z): d\left(f(y), T x_{0}^{\prime \prime}(y)\right)<\beta\right\}$. These two sets are open in $(C(Y, Z), p)$ and $T x_{0}^{\prime} \in V^{\prime}, T x_{0}^{\prime \prime} \in V^{\prime \prime}$. According to $p$-continuity of $T: X \rightarrow C(Y, Z)$ there exist two open neighborhoods $U^{\prime}$ and $U^{\prime \prime}$ of $x_{0}^{\prime}$ and $x_{0}^{\prime \prime}$ correspondingly, such that $T U^{\prime} \subset V^{\prime}$ and $T U^{\prime \prime} \subset V^{\prime \prime}$. Since $\gamma$ is an open covering and $X$ is a regular space, we may assume that the closures $\bar{U}^{\prime}, \bar{U}^{\prime \prime}$ are $\gamma$-small and lie in $U$. Thus (i) is fulfilled. To check (ii), take $x^{\prime} \in U^{\prime}, x^{\prime \prime} \in U^{\prime \prime}$. Then $T x^{\prime} \in V^{\prime}$ and $T x^{\prime \prime} \in V^{\prime \prime}$. In another words $d\left(T x^{\prime}(y), T x_{0}^{\prime}(y)\right)<\beta$ and $d\left(T x^{\prime \prime}(y), T x_{0}^{\prime \prime}(y)\right)<\beta$. Then $d\left(T x^{\prime}(y), T x^{\prime \prime}(y)\right) \geqq d\left(T x_{0}^{\prime}(y)\right.$, $\left.T x_{0}^{\prime \prime}(y)\right)-d\left(T x_{0}^{\prime}(y), T x^{\prime}(y)\right)-d\left(T x^{\prime \prime}(y), T x_{0}^{\prime \prime}(y)\right)>d\left(T x_{0}^{\prime}(y), T x_{0}^{\prime \prime}(y)\right)-$ $2 \beta=1 / n$. Lemma 1.4 is proved. 
Suppose that the set $H_{n}$ is dense in some open $U_{0} \subset X, U_{0} \neq \varnothing$. By Lemma 1.4 (with $U=U_{0}$ and $\gamma=\gamma_{1}$ ) there will exist two nonempty open sets $U_{00}:=U^{\prime} \subset U_{0}, U_{01}:=U^{\prime \prime} \subset U_{0}$ and $y_{0}:=y \in Y$ such that (i) and (ii) from Lemma 1.4 are satisfied. Since $H_{n}$ was supposed to be dense in $U_{0}, H_{n} \cap U_{00} \neq \varnothing$ and $H_{n} \cap U_{01} \neq \varnothing$. We can apply now Lemma 1.4 to $U_{00}$ and get as above a pair of nonempty open subsets $U_{000}, U_{001}$ of $U_{00}$ and a point $y_{00} \in Y$ such that (i) and (ii) from Lemma 1.4 are fulfilled for $U^{\prime}=U_{000}, U^{\prime \prime}=U_{001}, y=y_{00}$ and $\gamma=\gamma_{2}$. The same can be done with $U_{01}$ in order to get another pair of $\gamma_{2}$-small sets $U_{010}, U_{011} \subset U_{01}$ and a point $y_{01} \in Y$ such that (i) and (ii) from the lemma are satisfied. Proceeding by induction, we can construct a sequence of open nonempty subsets of $X$ and a sequence of points in $Y$ which are indexed by symbols of the type $j_{0} j_{1} j_{2} \cdots j_{k}$, where $j_{0}=0$ and all other $j_{i}, i=1,2, \cdots, k$, are either equal to 0 or to 1 . This is done in such a way that

(1) $\bar{U}_{j_{0} j_{1} j_{2} \cdots j_{k}}$ is $\gamma_{k}$-small subset of $X$;

(2) if $p<k$, then the closure $\bar{U}_{j_{0} j_{1} \cdots j_{k}} \subset U_{j_{0} j_{1} \cdots j_{p}}$.

(3) if $j_{i}=j_{i}^{\prime}, i=0,1,2, \cdots, s$, and $j_{s+1} \neq j_{s+1}^{\prime}$, then for every $x^{\prime} \in U_{j_{0} j_{1} \cdots j_{s} j_{s}+1 \cdots j_{m}}$ and every $x^{\prime \prime} \in U_{j_{0}^{\prime} j_{1} \cdots j_{s}^{\prime} j_{s}^{\prime}+{ }_{1} \cdots j_{n}^{\prime}}$ the inequality $d\left(T x^{\prime}\left(y_{j_{0} j_{1} \cdots j_{s}}\right), T x^{\prime \prime}\left(y_{j_{0} j_{1} \cdots j_{s}}\right)\right)>1 / n$ holds.

Now take an arbitrary sequence $s=\left(j_{i}\right)_{i \geqq 0}$ with $j_{0}=0$ and $j_{i}, i \geqq 1$, equal to 0 or 1 . It defines a decreasing sequence of closed sets $\bar{U}_{0}=\bar{U}_{j_{0}} \supset \bar{U}_{j_{0} j_{1}} \supset \bar{U}_{j_{0} j_{1} j_{2}} \ldots$ By (1) and by the strong countable completeness of $X$, the intersection $\Delta(s):=\bigcap\left\{\bar{U}_{j_{0} j_{1} \cdots j_{k}}: k \geqq 0\right\}$ is not empty. It follows from (2) that $\Delta(s)=\bigcap\left\{U_{j_{1} j_{2} \cdots j_{k}}: k \geqq 0\right\}$. For another sequence $s^{\prime}=\left(j_{i}^{\prime}\right)_{i \geqslant 0}$ with $j_{j}^{\prime}=0$ and $j_{i}^{\prime}=0,1$, we get another decreasing sequence of closed sets $\left(\bar{U}_{\dot{j}^{\prime} j_{1}^{\prime} \cdots j_{k}^{\prime}}\right)_{k \geqslant 0}$ and another set $\Delta\left(s^{\prime}\right) \neq \varnothing$. From (3) it follows that for every $x^{\prime} \in \Delta\left(s^{\prime}\right)$ and $x^{\prime \prime} \in \Delta\left(s^{\prime \prime}\right)$ with $s^{\prime} \neq s^{\prime \prime}$,

$$
d\left(T x^{\prime}\left(y_{j_{0}^{\prime} j_{1}^{\prime} \cdots j_{k}^{\prime}}^{\prime}\right), \quad T x^{\prime \prime}\left(y_{j_{0}^{\prime \prime} j_{1}^{\prime} \cdots j_{k}^{\prime \prime}}\right)\right)>1 / n
$$

where $k+1$ is the smallest positive integer with $j_{k+1}^{\prime} \neq j_{k+1}^{\prime \prime}$ (this means that the two " $y$ " in $\left(^{*}\right)$ are one and the same point of $Y$ ).

Since there are uncountably many different sequences $s$ of the above type, we get uncountably many different sets $\Delta(s)$. Their images $T \Delta(s)$ under $T: X \rightarrow C(Y, Z)$ will form an uncoutable system in $C(Y, Z)$ which is (due to $\left(^{*}\right)$ ) "very discrete" in $(C(Y, Z), u)$, i.e., $d\left(f^{\prime}, f^{\prime \prime}\right)>1 / n$ whenever $f^{\prime} \in T\left(s^{\prime}\right), f^{\prime \prime} \in T\left(s^{\prime \prime}\right)$ and $s^{\prime} \neq s^{\prime \prime}$. Put $\Delta=$ $\cup\{U(s): s$ runs over all possible sequences $s\}$ and denote by $Y_{1}$ the closure in $Y$ of the sequence of points $y_{j_{0} j_{1} \cdots j_{k}} \in Y$ constructed above. Consider the restriction mapping $r: C(Y, Z) \rightarrow C\left(Y_{1}, Z\right)$ and the uniform metric in $C\left(Y_{1}, Z\right)$ (we denote it again by $d(\cdot, \cdot)$ ). Then the system $\{r \cdot T \Delta(s): s$ runs over all possible sequences $\}$ will form in 
$\left(C\left(Y_{1}, Z\right), u\right)$ (due to $\left.\left(^{*}\right)\right)$ an uncountable discrete family. Therefore, it will be a contradiction and the proof of Theorem 1.1 will be finished, if we show that $r \circ T \Delta$ is a separable subset of $\left(C\left(Y_{1}, Z\right), u\right)$. The following lemma together with the requirement of Theorem 1.1 shows that this is the case.

LEMMA 1.5. The set $\Delta \subset X$ is countably compact.

This is almost trivial. For a given sequence $\left(x_{m}\right)_{m \cong 1} \subset \Delta$ we find step by step a sequence of zero's or one's $\left(j_{i}^{0}\right)_{i \geqslant 0}$ with $j_{0}^{0}=0$, such that each set $U_{j \cap j 1 \ldots j k}^{00}$ from the construction of $\Delta$ contains infinitely many members of the sequence $\left(x_{m}\right)_{m \geq 1}$. The set $F_{k}=\overline{U_{j_{0}^{0} j_{1}^{0} \cdots j_{s}^{0} \cap}}$ $\overline{\left(x_{m}\right)_{m \geqq k}}$ is then closed, nonempty and $\gamma_{k}$-small. Therefore $\bigcap_{k \geqq 1} F_{k} \neq \varnothing$. Every point from this nonempty intersection is a cluster point for the sequence $\left(x_{m}\right)_{m \smile 1}$. Lemma 1.5 is proved. Theorem 1.1 is also proved.

Corollary 1.6. (Namioka [13], Theorem 2.2). Let $X$ be a strongly countably complete regular space, let $Y$ be a compact space and $Z$ be a metric space. If $T: X \rightarrow(C(Y, Z), p)$ is a continuous map, then there exists a dense $G_{-}$-set $A$ in $X$ such that, at each point of $A, T$ is u-continuous.

Proof. Apply Theorem 1.1. This is possible because of Proposition 1.2 .

Corollary 1.7. (Namioka [13], Theorem 4.1). Let $X$ be a strongly countably complete regular space and let $E$ be a normed space. If $T: X \rightarrow(E$, weak $)$ is continuous, then there is a dense $G_{-}$-set $A$ in $X$ such that, at each point of $A$, the map $T$ is norm continuous. ${ }^{1}$

Proof. Denote by $B^{*}$ the unit ball of the dual space $E^{*}$ endowed with the weak*-topology. The space $E$ can be thought of as a subset of $C\left(B^{*}, R\right)$. The pointwise convergence topology $p$ of $C\left(B^{*}, R\right)$ generates the weak topology in $E$ and the norm topology in $E$ is just the one inherited by $\left(C\left(B^{*}, R\right), u\right)$. Since $B^{*}$ is weak*compact space, it remains to apply 1.6 to the map $T: X \rightarrow E \subset$ $C\left(B^{*}, R\right)$.

Corollary 1.8. (Namioka [13], Corollary 4.2). Let $K$ be a weak-compact subset of the normed space $E$. Then there is a dense

${ }^{1}$ For separable normed spaces $E$ this result belongs to Alexiewicz and Orlicz [1; see also Fort [7]. 
$G_{o}$-set $A$ in $(K$, weak) such that the identity map (K, weak) $\rightarrow$ $(K$, norm $)$ is continuous at each point of $A$.

Proof. ( $K$, weak) is compact and thus strongly countably complete.

COROLlaRy 1.9. Let every separable closed linear subspace of the Banach space $E$ have a separable dual and let $X$ be a strongly countably complete regular space. If $T: X \rightarrow E^{*}$ is a weak*-continuous mapping, then $T$ is norm-continuous at the points of some dense $G_{i}$-subset of $X$.

Proof. Proposition 1.3 shows that Theorem 1.1 can be applied.

CoROLlaRY 1.10. Let every separable closed linear subspace of the Banach space $E$ have a separable dual and let $K^{*}$ be a weak*compact subset of $E^{*}$. Then there is a dense $G_{i}$-subset $A$ of $\left(K^{*}, w^{*}\right)$ such that, at every point of $A$, the identity mapping $\left(K^{*}, w^{*}\right) \rightarrow$ $\left(K^{*}, n^{*}\right)$ is continuous.

Proof. This is immediate from Corollary 1.9 because $\left(K^{*}, w^{*}\right)$ is a compact and thus a strongly countably complete space.

REMARK 1.11. It follows from Corollary 1.10 that, if every separable subspace of the Banach space $E$ has a separable dual, then every weak*-compact set $K^{*}$ of $E^{*}$ contains relatively weak*open subsets with arbitrarily small diameter. This property in turn (see the proof of Proposition 9 from the paper of Namioka and Phelps [14]) implies that every separable linear subspace of $E$ has a separable dual. Therefore the assertion contained in Corollary 1.9 is actually nessesary and sufficient condition for the space $E$ to be an Asplund space (see also Lemma 3 and Corollary 10 from Namioka and Phelps [14]).

2. Cantor-set-construction for multivalued mappings. We want to give here another version of Theorem 1.1 which concerns multivalued maps. Considered for single-valued mappings only, the "multivalued result" does not give the full generality of Theorem 1.1. But it is still sufficiently general to provide some new and useful information concerning continuity properties of monotone mappings and metric projections. As corollaries we get also new proofs of some known and important results about the properties of weak-compact subsets of Banach spaces and about differentiability of convex functions defined on such spaces. 
Fist of all we recall some definitions and notations. The multivalued map $T: X \rightarrow Y$, where $X, Y$ are topological spaces, is said to be upper semicontinuous (usc) at some point $x_{0} \in X$ if, for every open set $U \subset Y$ with $T x_{0} \subset U$, there exists a neighborhood $V$ of $x_{0}$ in $X$ such that $T x \subset U$ for each $x$ from $V$. The mapping $T: X \rightarrow Y$ is called upper semicontinuous if it is upper semicontinuous at every point of the space $X$.

Let $(F,\|\cdot\|)$ be a normed space with unit ball $B$. Denote the unit ball of the dual space $F^{*}$ by $B^{*}$. Take $Y \subset B^{*}$ and put $Y_{\perp}=\{f \in F:\langle f, y\rangle=0$ for every $y \in Y\}$. The quotient space $Q(Y)=$ $F / Y_{\perp}$ is also a normed space. The elements of $Y$ can be viewed as continuous linear functionals on $(Q(Y),\|\cdot\|)$ and it is not difficult to see that, for each $q \in Q(Y)$, the norm $\|q\|$ is greater than or equal to $\sup \{|\langle q, y\rangle|: y \in Y\}$ (for any $f \in F,\|f\|=\sup \left\{\left|\left\langle f, f^{*}\right\rangle\right|: f^{*} \in B^{*}\right\} \geqq$ $\sup \{|\langle f, y\rangle|: y \in Y\} ;$ if $1 \in Y_{\perp}$, then $\|f+1\| \geqq \sup \{\mid\langle f, y\rangle: y \in Y\}$; this means that the norm of $q=f+Y_{\perp}$ in $Q(Y)$ satisfies the above inequality).

Further, the linear subspace $G$ of $F^{*}$ will be called "norming subspace" if

$$
\|f\|=\sup \left\{\langle f, g\rangle: g \in G \cap B^{*}\right\} \text { for each } f \text { from } F .
$$

By $w(F, G)$ (resp. $w(G, F)$ ) we will denote the weakest topology in $F$ (resp. in $G$ ) with respect to which all elements of $G$ (resp. of $F$ ) regarded as linear functionals on $F$ (resp. on $G$ ) are continuous.

For a given $Y \subset B^{*}$ we denote by $r_{Y}$ the quotient mapping $F \rightarrow$ $Q(Y)=F / Y_{\perp}$.

TheOREM 2.1. Let $X$ be a strongly countably complete space, $(F,\|\cdot\|)$ be a normed space and $G$ be a norming subspace of $F^{*}$. Suppose $T: X \rightarrow(F, w(F, G)$ ) is an upper semicontinuous (multivalued) mapping with $w(F, G)$-compact and convex images and such that for every countably compact $\Delta \subset X$ and every countable $Y \subset B^{*} \cap G$, the set $r_{Y} \circ T(\Delta)$ is separable in $(Q(Y),\|\cdot\|)$. Then there exists a dense $G_{i}$-set $A$ in $X$ at every point $x$ of which the following "continuity property" (cp) is fulfilled:

$(c p)$

for every $\varepsilon>0$ there is an open $U \subset X, U \ni x$, such that $\inf \left\{\left\|f^{\prime}-f^{\prime \prime}\right\|: f^{\prime} \in T x^{\prime}, f^{\prime \prime} \in T x^{\prime \prime}\right\} \leqq \varepsilon$ whenever $x^{\prime}, x^{\prime \prime} \in U$.

As in $\S 1$ we will first point out two situations in which all requirements of this theorem are fulfilled. This situations are important for us because of the applications we have in mind. As a first step we need a topological result. 
Lemma 2.2. Suppose $T: M_{1} \rightarrow M_{2}$ is an upper semicontinuous mapping with countably compact images from one topological space $M_{1}$ into another $M_{2}$. If $\Delta \subset M_{1}$ is countably compact, then $T(\Delta)=$ $\bigcup\{T x: x \in \Delta\}$ is also a countably compact subset of $M_{2}$.

Proof. Let $s=\left(y_{i}\right)_{i \geqq 1}$ be a sequence in $T(\Delta)$ and let $x_{i} \in \Delta, i=$ $1,2, \cdots$, be such points that $y_{i} \in T x_{i}$. Consider the closures $\overline{\left(y_{i}\right)_{i \geq k}}$ of the tails of the sequence $\left(y_{i}\right)_{i \geq 1}$. It is enough to show that the set $\left.\cap \overline{\left\{\left(y_{i}\right)_{i \geqq 1}\right.}: k \geqq 1\right\}$ is not empty. Since $\Delta$ is a countably compact subset of $M_{1}$, we can find a point $\bar{x} \in \cap\left\{\overline{\left(x_{i}\right)_{i \geqq k}}: k \geqq 1\right\}$. The set $T \bar{x}$ is countably compact and the proof will be finished if we show that $T \underline{x} \cap \overline{\left(y_{i}\right)_{i \geqq k}}$ is not empty for every $k \geqq 1$. Suppose this is not the case. By the upper semicontinuity of $T$ at $\bar{x}$ we can find an open $V \subset M_{1}, \bar{x} \in V$, such that $T(V) \cap \overline{\left(y_{i}\right)_{i \geqq k}}=\varnothing$. But this contradicts the fact that $V$, as an open neighborhood of the cluster point $\bar{x}$, must contain $x_{i}$ with arbitrarily large indices.

Proposition 2.3. Let $\Delta$ be a countably compact space, $(F,\|\cdot\|)$ be a normed space and $T: \Delta \rightarrow(F$, weak) be an upper semicontinuous mapping with weak-compact inages $T x, x \in \Delta$. Then, for every countable $Y \subset F^{*}$, the set $r_{Y} \circ T(\Delta)$ is a norm-separable subset of $Q(Y)$. In other words, Theorem 2.1 is valid in the case when G coincides with all of $F^{*}$.

Proof. Since the quotient map $r_{Y}: F \rightarrow Q(Y)$ is continuous with respect to the weak topologies in $F$ and $Q(Y)$, it follows from the previous lemma that $r_{Y} \circ T(\Delta)$ is a weak-countably compact subset of the normed space $Q(Y)$. On the other hand, the countable set $Y$, which can be thought of as a subset of $Q(Y)^{*}$, generates a metric topology " $m$ " in $Q(Y)$ which is weaker than the weak topology of $Q(Y)$. The set $r_{Y} \circ T(\Delta)$ will be $m$-countably compact and hence $m$-compact. As any other metrizable compact, the space $\left(r_{Y} \circ T(\Delta), m\right)$ is separable and has a countable dence subset $C \subset r_{Y} \circ T(\Delta)$. As in the proof of Proposition 1.2 we can prove that $C$ is dence in $r_{Y} \circ T(\Delta)$ in the space $\left(Q(Y)\right.$, weak). Indeed, for $q_{0} \in r_{Y} \circ T(\Delta)$ we can find a sequence $\left(q_{i}\right)_{i \geqq 1} \subset C$ which is $m$-converging to $q_{0}$. As $r_{Y}^{\circ} \circ T(\Delta) \supset C$ is weak-countably compact, the sequence $\left(q_{i}\right)_{i \geqq 1}$ must have a weakcluster point $\bar{q} \in r_{Y} \circ T(\Delta)$. Then $\bar{q}$ is also $m$-cluster point of the sequence $\left(q_{i}\right)_{i \geqq 1}$. Since the latter sequence has only one $m$-cluster point and this is $q_{0}$, we get $\bar{q}=q_{0}$. This means that $r_{Y} \circ T(\Delta)$ is a weak separable subset of $Q(Y)$. Therefore it will be also normseparable subset of $Q(Y)$. The proposition is proved.

Proposition 2.4. Let $E$ be a normed space such that every 
separable subspace of it has a separable dual. Put $(F,\|\cdot\|):=$ $\left(E^{*}\right.$, norm $\left.^{*}\right)$ and $G:=E \subset E^{* *}$. Then the requirements of Theorem 2.1 are fulfilled for every upper semicontinuous mapping $T: X \rightarrow$ $(F, w(F, G))$ with convex and $w(F, G)$-compact images.

The proof can be omitted.

Proof of Theorem 2.1. Let $n$ be a positive integer and $H_{n}$ be the set of all $x \in X$ such that in every open $U \subset X, x \in U$, there exist at least one pair $x^{\prime}, x^{\prime \prime} \in U$ with $\inf \left\{\left\|f^{\prime}-f^{\prime \prime}\right\|: f^{\prime} \in T x^{\prime}, f^{\prime \prime} \in\right.$ $\left.T x^{\prime \prime}\right\}>1 / n$. As in the proof of Theorem 1.1 we need a lemma.

LEMMA 2.5. Let $y$ be an open covering of $X$ and $U$ be an open subset of $X$ with $U \cap H_{n} \neq \varnothing$. Then there exist two nonempty open sets $U^{\prime}, U^{\prime \prime}$ and an element $g \in G \cap B^{*}$ such that

(i) the closures $\bar{U}^{\prime}, \bar{U}^{\prime \prime}$ in $X$ of $U^{\prime}, U^{\prime \prime}$ are contained in $U$ and are $\gamma$-small subsets of $X$;

(ii) $\left|\left\langle f^{\prime}-f^{\prime \prime}, g\right\rangle\right|>1 / n$ whenever $f^{\prime} \in T x^{\prime}, f^{\prime \prime} \in T x^{\prime \prime}$ and $x^{\prime} \in U^{\prime}$, $x^{\prime \prime} \in U^{\prime \prime}$.

Proof of the lemma. As $U \cap H_{n} \neq \varnothing$, there exists a pair $x_{0}^{\prime}$, $x_{0}^{\prime \prime} \in U$ with $\inf \left\{\left\|f^{\prime}-f^{\prime \prime}\right\|: f^{\prime} \in T x_{0}^{\prime}, f^{\prime \prime} \in T x_{0}^{\prime \prime}\right\}>1 / n$. Since the images $T x$ are convex and compact and since $G$ is a norming subspaces of $F^{*}$ we can find some $g \in B^{*} \cap G$ such that the number $a$ : = $\min \left\{\left\langle f^{\prime}, g\right\rangle: f^{\prime} \in T x_{0}^{\prime}\right\}-\max \left\{\left\langle f^{\prime \prime}, g\right\rangle: f^{\prime \prime} \in T x_{0}^{\prime \prime}\right\}$ is greater than $1 / n$. Put $\beta:=(a-(1 / n)) / 2$ and consider the sets $V^{\prime}$ and $V^{\prime \prime}$, where $V^{\prime}=$ $\left\{f \in F:\langle f, g\rangle>\min \left\{\left\langle f^{\prime}, g\right\rangle: f^{\prime} \in T x_{0}^{\prime}\right\}-\beta\right\}$ and $V^{\prime \prime}=\{f \in F:\langle f, g\rangle<$ $\left.\max \left\{\left\langle f^{\prime \prime}, g\right\rangle: f^{\prime \prime} \in T x_{0}^{\prime \prime}\right\}+\beta\right\}$. These two sets are $w(F, G)$-open in $F$ and $T x_{0}^{\prime} \subset V^{\prime}, T x_{0}^{\prime \prime} \subset V^{\prime \prime}$. Since $T: X \rightarrow(F, w(F, G))$ is upper semicontinuous at $x_{0}^{\prime}$ and $x_{0}^{\prime \prime}$, we find two open neighborhoods $U^{\prime}$ of $x_{0}^{\prime}$ and $U^{\prime \prime}$ of $x_{0}^{\prime \prime}$ such that $T x^{\prime} \subset V^{\prime}$ for every $x^{\prime} \in U^{\prime}$ and $T x^{\prime \prime} \subset V^{\prime \prime}$ for every $x^{\prime \prime} \in U^{\prime \prime}$. Since $\gamma$ is an open covering of the regular space $X$, we may assume that the sets $\bar{U}^{\prime}$ and $\bar{U}^{\prime \prime}$ are $\gamma$-small and lie in $U$. Thus (i) is proved. To check (ii), we take any $x^{\prime} \in U^{\prime}$ and $x^{\prime \prime} \in U^{\prime \prime}$. Because of $T x^{\prime} \subset V^{\prime}$ and $T x^{\prime \prime} \subset V^{\prime \prime}$, we have, for every $f^{\prime} \in T x^{\prime}, f^{\prime \prime} \in T x^{\prime \prime},\left\langle f^{\prime}-f^{\prime \prime}, g\right\rangle>\min \left\{\left\langle f^{\prime}, g\right\rangle: f^{\prime} \in T x_{0}^{\prime}\right\}-\max \left\{\left\langle f^{\prime \prime}, g\right\rangle:\right.$ $\left.f^{\prime \prime} \in T x_{0}^{\prime \prime}\right\}-2 \beta=a-2 \beta=1 / n$. Lemma 2.5 is proved.

Exactly as in the proof of Theorem 1.1 we construct (with the help of Lemma 2.5) a sequence $\left(g_{j}\right)_{j \geqq 1}$ in $B^{*} \cap G$ and an uncountable subset $X_{1}$ of $X$ such that

(a) $X_{1}$ is a subset of some countably compact $\Delta \subset X$.

(b) for every $x^{\prime}, x^{\prime \prime} \in X_{1}, x^{\prime} \neq x^{\prime \prime}$, there exists $g \in\left(g_{j}\right)_{j \geqq 1}$ for which 
$\left.{ }^{* *}\right) \quad \min \left\{\left\langle f^{\prime}, g\right\rangle: f^{\prime} \in T x^{\prime}\right\}-\max \left\{\left\langle f^{\prime \prime}, g\right\rangle: f^{\prime \prime} \in T x^{\prime \prime}\right\}>1 / n$.

Put $Y_{1}=\left(g_{j}\right)_{j \geq 1}$ and consider the quotient space $\left(Q\left(Y_{1}\right),\|\cdot\|\right)$ and its subset $r_{Y_{1}} \circ T\left(X_{1}\right)$. By the hypothesis of Theorem 2.1 the latter set must be norm-separable. On the other hand, by $\left({ }^{* *}\right)$ this set contains an uncountable "discrete" subset $X_{1}$. This contradiction proves the theorem.

We now give some applications of this theorem.

Let $(E,\|\cdot\|)$ be a Banach space which is generated by some weakly-compact set $K \subset E$ (i.e., the linear span $L(K)$ of $K$ in $E$ is norm dense in $E$ ). Without loss of generality we may assume that $K$ is convex and symmetric. Consider in $E^{*}$ the support function of $K: h\left(e^{*}\right)=\max \left\{\left\langle e, e^{*}\right\rangle: e \in K\right\}$. It is a nonnegative, convex and positively homogeneous function. Because $L(K)$ is dence in $(E, \| \cdot \mid)$, it follows from $h\left(e^{*}\right)=0$ that $\left\langle e, e^{*}\right\rangle=0$ for every $e \in E$. This means that $\left(E^{*}, h(\cdot)\right)$ is a normed space with norm $h(\cdot)$.

Proposition 2.6. The dual of $\left(E^{*}, h().\right)$ is exactly $L(K)$.

Proof. Let $s\left(e^{*}\right)$ be a continuous linear functional in $\left(E^{*}, h().\right)$. Then $s($.$) will be continuous relative the norm topology of E^{*}$. Hence $s \in E^{* *}$. It is no restriction to assume that the supremum of $s\left(e^{*}\right)$ on the unit ball of the norm $h($.$) in E^{*}$ is less or equal to 1. Since the latter unit ball is just the polar of $K$ in $E^{*}, s$ will belong to the second polar of $K$ (taken in $E^{* *}$ ). By the theorem on bipolars $s$ must belong to the closure of $K$ in $\left(E^{* *}, w\left(E^{* *}, E^{*}\right)\right)$. But $K$ is $w\left(E, E^{*}\right)$-compact subset of $E$ and is already $w\left(E^{* *}, E^{*}\right)$-closed in $E^{* *}$. Therefore $s$ will belong to $K$. Proposition 2.6 is proved.

Definition 2.7. The (multivalued) mapping $T: E \rightarrow E^{*}$ from the Banach space into its dual $E^{*}$ is called monotone if $\left\langle x_{1}-x_{2}, x_{1}^{*}-x_{2}^{*}\right\rangle \geqq 0$ whenever $x_{i}^{*} \in T x_{i}, i=1,2 . T$ is said to be a maximal monotone mapping from $E$ to $E^{*}$ if its graph is not properly contained into the graph of any other monotone mapping from $E$ to $E^{*}$. Using Zorn's lemma it is not difficult to see that the graph of every monotone mapping $T$ is contained into the graph of some maximal monotone mapping. $D(T)$ will donote the set $\{x \in E: T x \neq \varnothing\}$ and int $D(T)$ will stand for the norm interior of the set $D(T) \subset E$. Using some results of Rockafellar [15] and Browder [6] it is easy to prove (see Kenderov [9]) that every maximal monotone mapping $T$ : int $D(T) \rightarrow\left(E^{*}, w\left(E^{*}, E\right)\right)$ is an upper semiconinuous mapping with convex and $w\left(E^{*}, E\right)$-compact images.

THEOREM 2.8. Let $E$ be a Banach space generated by the weak- 
compact, symmetric and convex set $K$. Denote the support function of $K$ by $h($.$) and suppose T: E \rightarrow E^{*}$ is a monotone mapping. Then there exists a dense $G_{i}$-subset $A$ of (int $D(T),\|\cdot\|$ ) such that, for each $x$ from $A$,

(i) $T x$ is a singleton.

(ii) $T:(E,\|\cdot\|) \rightarrow\left(E^{*}, h(\cdot)\right)$ is upper semicontinuous at $x$.

Proof. Without any harm $T$ can be taken to be a maximal monotone mapping. Then $T:(E,\|\cdot\|) \rightarrow\left(E^{*}, w\left(E^{*}, L(K)\right)\right)$ is an upper semicontinuous mapping with convex and $w\left(E^{*}, L(K)\right)$-compact images. Put $X=(\operatorname{int} D(T),\|\cdot\|), F=\left(E^{*}, h(\cdot)\right)$ and $G=L(K)$. Propositions 2.3 and 2.6 indicate that Theorem 2.1 can be applied to the mapping $T: X \rightarrow F$. This yields a dense $G_{i}$-subset $A$ of (int $D(T),\|\cdot\|)$ such that, for every $x_{0} \in A$, the following is fulfilled:

(cp) $\left\{\begin{array}{l}\text { for every } \varepsilon>0 \text { there exists an open } U \subset X, U \ni x_{0} \text {, with } \\ \inf \left\{h\left(e^{\prime *}-e^{\prime \prime *}\right): e^{\prime *} \in T x^{\prime}, e^{\prime \prime *} \in T x^{\prime \prime}\right\} \leqq \varepsilon \text { whenever } x^{\prime}, x^{\prime \prime} \in U .\end{array}\right.$

The following lemma completes the proof and shows how the $(c p)$-condition can be used in order to prove that $T$ is single-valued and upper semicontinuous at $x_{0} \in A$ in the desired sense.

LEMMA 2.9. If $x_{0}$ belongs to the above determined set $A \subset$ $\operatorname{int} D(T)$, then $T x_{0}$ contains only one point and $T:(E,\|\cdot\|) \rightarrow$ $\left(E^{*}, h(\cdot)\right)$ is upper semicontinuous at $x_{0}$.

Proof. It suffices to show that the $h$-diameter of the set $T U$ is less or equal to $2 \varepsilon$. We prove this by showing that, whatever $e_{1}^{*}, e_{2}^{*} \in T U$ and $e \in K,\left\langle e, e_{1}^{*}-e_{2}^{*}\right\rangle\left\langle 2 \varepsilon\right.$. Let $e \in K, e \neq 0$, and $e_{i}^{*} \in T x_{i}$, $x_{i} \in U, i=1,2$. When the number $t>0$ is small enough, the points $x^{\prime}=x_{1}+t e$ and $x^{\prime \prime}=x_{2}-t e$ still belong to the open set $U \subset \operatorname{int} D(T)$. By $(c p)$ there exist $e^{\prime *} \in T x^{\prime}, e^{\prime \prime *} \in T x^{\prime \prime}$ with $h\left(e^{\prime *}-e^{\prime * *}\right)=\sup \left\{\left\langle z, e^{\prime *}-\right.\right.$ $\left.\left.e^{\prime * *}\right\rangle: z \in K\right\}<2 \varepsilon$. In particular

(a) $\left\langle e, e^{\prime *}-e^{\prime \prime *}\right\rangle\langle 2 \varepsilon$.

By the monotonicity of $T$ (with the inequality applied for the points $x^{\prime}$ and $x_{1}$ ) we get

(b) $\left\langle e, e^{*}-e_{1}^{*}\right\rangle \geqq 0$.

Again by the monotonicity (for the points $x^{\prime \prime}, x_{2}$ )

(c) $\left\langle e, e_{2}^{*}-e^{\prime \prime *}\right\rangle \geqq 0$.

The three inequalities (a), (b) and (c) give $\left\langle e, e_{1}^{*}-e_{2}^{*}\right\rangle=\left\langle e, e_{1}^{*}-e^{*}\right\rangle+$ $\left\langle e, e^{\prime *}-e^{\prime *}\right\rangle+\left\langle e, e^{\prime *}-e_{2}^{*}\right\rangle \leqq\left\langle e, e^{\prime *}-e^{\prime * *}\right\rangle\langle 2 \varepsilon$. Theorem 2.8 is proved.

REMARK 2.10. That every monotone mapping $T: E \rightarrow E^{*}$, defined on the weak-compactly generated Banach space $E$, must be 
single-valued at the points of some dense $G_{\delta}$-subset of int $D(T)$ was proved in another way in Kenderov $[9,10]$. We obtained here additional information contained in (ii) of 2.9.

Let now $f: E \rightarrow R$ be a continuous real-valued function. It is called Gateaux differentiable at some $x_{0} \in E$ if there exists $x_{0}^{*} \in E^{*}$ such that $\lim _{t \rightarrow 0}\left(f\left(x_{0}+t e\right)-f\left(x_{0}\right)\right) / t=\left\langle e, x_{0}^{*}\right\rangle$ for every $e \in E$. When $K$ is a bounded subset of $E$, the function $f$ is said to be $K$-smooth (Borwein [4]) at $x_{0}$ if it is Gateaux differentiable at $x_{0}$ and $\left|\left(\left(f\left(x_{0}+t e\right)-f\left(x_{0}\right)\right) / t\right)-\left\langle e, x_{0}^{*}\right\rangle\right|$ tends to zero uniformly for $e$ in $K$ as $t \rightarrow 0$. If $K$ is the unit ball of $E$ and $f$ is $K$-smooth at $x_{0}$, then $f$ is called Frechet differentiable at $x_{0}$.

COROLlaRy 2.11. (Borwein [4], Theorem 2.2). Let the Banach space $E$ be generated by the weakly compact set $K \subset E$. Then every continuous convex function $f: E \rightarrow R$ is $K$-smooth at the points of some dense $G_{\hat{o}}$-subset of $(E,\|\cdot\|)$. In particular (see Asplund [2] and Trojansky [19] for another proof), if $E$ is a reflexive space, then $f$ is Frechet differentiable at the points of some dense $G_{-}$-subset of $E$.

Proof. The subgradient $\partial: E \rightarrow E^{*}$ of the convex and continuous function $f$ is a multivalued monotone mapping assigning to each $x_{0} \in E$ the nonempty set $\partial\left(x_{0}\right)=\left\{x^{*} \in E^{*}: f(x)-f\left(x_{0}\right) \geqq\left\langle x-x_{0}, x^{*}\right\rangle\right.$ for every $x \in E\}$. Since $f$ is Gateaux differentiable at $x_{0}$ precisely when $\partial\left(x_{0}\right)$ consists of only one point, it follows from Theorem 2.8(i) that there exists a dense $G_{i}$-subset $A \subset E$ at each point of which $f$ is Gateaux differentiable (for another proof of the last assertion see Asplund [2], Theorem 2). According to a known result (see Asplund and Rockafellar [3]) the condition (ii) of Theorem 2.8 implies that, at each point $x_{0}$ of $A$, the function $f$ is $K$-smooth. For the sake of completeness and to meet our needs in the following pages we give a short proof of the auxiliary result. With no harm $K$ can be supposed symmetric and convex. By $h(\cdot)$ we denote again the support function of $K$.

Lemma 2.12. If the subgradient $\partial:(E,\|\cdot\|) \rightarrow\left(E^{*}, h(\cdot)\right)$ is singlevalued and upper semicontinuous at some $x_{0} \in E$, then $f$ is $K$-smooth at $x_{0}$.

Proof. Let $e \in K$ and $t$ be a number. Denote by $x_{t}^{*}$ some point of $\partial\left(x_{0}+t e\right)$ and by $x_{0}^{*}$ the point $\partial\left(x_{0}\right)$. According to the definition of $\partial$ we have $f\left(x_{0}+t e\right)-f\left(x_{0}\right) \geqq\left\langle t e, x_{0}^{*}\right\rangle$ and $f\left(x_{0}\right)-f\left(x_{0}+t e\right) \geqq$ $\left\langle-t e, x_{t}^{*}\right\rangle$. Then $0 \leqq f\left(x_{0}+t e\right)-f\left(x_{0}\right)-\left\langle t e, x_{0}^{*}\right\rangle \leqq\left\langle t e, x_{t}^{*}-x_{0}^{*}\right\rangle$. Therefore $\left|\left(\left(f\left(x_{0}+t e\right)-f\left(x_{0}\right)\right) / t\right)-\left\langle e, x_{0}^{*}\right\rangle\right| \leqq\left|\left\langle e, x_{t}^{*}-x_{0}^{*}\right\rangle\right| \leqq h\left(x_{t}^{*}-x_{0}^{*}\right)$. 
By the upper semicontinuity of $\partial:(E,\|\cdot\|) \rightarrow\left(E^{*}, h(\cdot)\right)$ at $x_{0}, h\left(x_{t}^{*}-x_{0}^{*}\right)$ can be made arbitrarily small (when $t \rightarrow 0$ ) independently of $e \in K$. Theorem 2.11 is proved.

In the next application of Theorem 2.1 the set $K$ will play a role quite different from the role it was playing till now.

DEFinition 2.13. Let $K$ be a subset of the Banach space $E$, $e^{*} \in E^{*}$ and $t>0$. Set once more $h\left(e^{*}\right)=\sup \left\{\left\langle e, e^{*}\right\rangle: e \in K\right\}$. The set $\left.S\left(K, e^{*}, t\right)=\left\{e \in K:\left\langle e, e^{*}\right\rangle\right\rangle h\left(e^{*}\right)-t\right\}$ is called a slice of $K$ generated by $e^{*} \in E^{*}$ and $t>0$. The point $e_{0} \in K$ is said to be a strongly exposed point of $K$ if there exists some $e^{*} \in E^{*}$ for which $\left\langle e_{0}, e^{*}\right\rangle=h\left(e^{*}\right)$ and $\lim _{t \rightarrow 0} \operatorname{diam} S\left(K, e^{*}, t\right)=0$. It is said in this case that $e^{*}$ strongly exposes the point $e_{0}$ of $K$ or, simply, that $e^{*}$ strongly exposes $K$.

THEOREM 2.14. (Bourgain [5]). Let $K$ be a weakly compact subset of the Banach space $E$. Then there is a dense $G_{i}$-set $A$ in $\left(E^{*},\|\cdot\|\right)$ such that each $e_{0}^{*} \in A$ strongly exposes $K$.

Proof. Define a (multivalued) mapping $T: E^{*} \rightarrow K \subset E$ by the rule: $T\left(e^{*}\right)=\left\{e \in K:\left\langle e, e^{*}\right\rangle=h\left(e^{*}\right)\right\}$. It is not difficult to see that the graph of $T$ is closed in $\left(E^{*},\|\cdot\|\right) \times(K$, weak). Since $(K$, weak) is a compact space, the mapping $T:\left(E^{*},\|\cdot\|\right) \rightarrow(E$, weak $)$ is upper semicontinuous. Put $X:=\left(E^{*},\|\cdot\|\right), F:=(E,\|\cdot\|)$ and $G:=E^{*}$. Apply Theorem 2.1 (this is possible by Proposition 2.3). This yields a dense $G_{i^{-}}$-subset $A$ of $\left(E^{*},\|\cdot\|\right)$ for each point $e_{0}^{*} \in A$ of which the following is true:

for every $\varepsilon>0$ there is $\delta>0$ such that $\left\|e_{0}^{*}-e^{* *}\right\|<\delta$ and $\left\|e_{0}^{*}-e^{\prime \prime *}\right\|<\delta$ imply $\inf \left\{\left\|f^{\prime \prime}-f^{\prime}\right\|: f^{\prime} \in T e^{\prime *}, f^{\prime \prime} \in\right.$ $\left.T e^{\prime *}\right\} \leqq \varepsilon$.

From this "continuity property" we will deduce now that every $e_{0}^{*} \in A$ strongly exposes $K$. Indeed, take a slice $S:=S\left(K, e_{0}^{*}, t\right)$, where $t>0$. We have to show that the diameter of $S$ in $(E,\|\cdot\|)$ tends to 0 as $t \rightarrow 0$. To do this we take any $x \in S, e_{0} \in T e_{0}^{*}, e^{*} \in E^{*}$ and estimate $\left|\left\langle x-e_{0}, e^{*}\right\rangle\right|$.

Let $f^{\prime} \in T\left(e_{0}^{*}+e^{*}\right)$ and $f^{\prime \prime} \in T\left(e_{0}^{*}-e^{*}\right)$. Then $\left\langle x-f^{\prime}, e^{*}\right\rangle=$ $\left\langle x-f^{\prime}, e^{*}+e_{0}^{*}\right\rangle+\left\langle f^{\prime}-e_{0}, e_{0}^{*}\right\rangle+\left\langle e_{0}-x, e_{0}^{*}\right\rangle$. The first two terms of the right-hand-side are less or equal to 0 by the very definition of the mapping $T$. Since, in addition, $x$ belongs to the slice $S$, we have

$$
\left\langle x-f^{\prime}, e^{*}\right\rangle<t .
$$

On the other hand $\left\langle f^{\prime \prime}-e_{0}, e^{*}\right\rangle=\left\langle e_{0}-f^{\prime \prime}, e_{0}^{*}-e^{*}\right\rangle+\left\langle f^{\prime \prime}-e_{0}, e_{, j}^{*}\right\rangle$. 
Again by the definition of $T$ the right-hand-side is $\leqq 0$ :

$$
\left\langle f^{\prime \prime}-e_{0}, e^{*}\right\rangle \leqq 0 \text {. }
$$

From (1) and (2) we get

$$
\left\langle x-e_{0}, e^{*}\right\rangle\left\langle t+\left\langle f^{\prime}-f^{\prime \prime}, e^{*}\right\rangle \leqq t+\left\|f^{\prime}-f^{\prime \prime}\right\| \cdot\left\|e^{*}\right\|\right. \text {. }
$$

Given $\varepsilon>0$, by the last $(c p)$ we find the corresponding $\delta>0$. For an arbitrary $u^{*} \in E^{*}$ with $\left\|u^{*}\right\|=1$ put $e^{*}=\delta u^{*}$ and choose $f^{\prime} \in$ $T\left(e_{0}^{*}+e^{*}\right)$ and $f^{\prime \prime} \in T\left(e_{0}^{*}-e^{*}\right)$ in such a manner that $\left\|f^{\prime}-f^{\prime \prime}\right\|<2 \varepsilon$. Then, for $0<t<\varepsilon \delta$, we have by $(3)\left\langle x-e_{0}, \delta u^{*}\right\rangle<t+\delta\left\|f^{\prime}-f^{\prime \prime}\right\|<$ $3 \varepsilon \delta$. This means that $\left\|x-e_{0}\right\| \leqq 3 \varepsilon$. Theorem 2.14 is proved.

We add one more remark. It follows from the proof that $T e_{0}^{*}$ is a singleton and that $T:\left(E^{*},\|\cdot\|\right) \rightarrow(K,\|\cdot\|) \subset(E,\|\cdot\|)$ is upper semicontinuous at $e_{0}^{*}$.

Now it is clear how to prove the following result.

THEOREM 2.15. (Lindenstrauss-Trojanski). Every weakly compact subset $K$ of the Banach space $E$ is the closed convex hull of its strongly exposed points.

THEOREM 2.16. (Kenderov [12]). Let E be an Asplund space and $T: E \rightarrow E^{*}$ be a monotone mapping. Then $T$ is single-valued and upper senicontinuous (with repect to the norm topologies in $E$ and $\left.E^{*}\right)$ at the points of some dense $G_{i}$-subset of (int $\left.D(T),\|\cdot\|\right)$.

Proof. In [12] the author proved this theorem using the fact that $E$ is an Asplund space if and only if every bounded subset of $E^{*}$ is dentable. We prove now the same theorem taking as a starting point the fact (see Uhl [20] and Stegall 117]) that $E$ is an Asplund space if and only if every separable linear subspace of $E$ has a separable dual.

It suffices to consider only maximal monotone mappings. Then, as pointed out after Definition 2.7, the mapping $T:(E,\|\cdot\|) \rightarrow\left(E^{*}\right.$, weak $^{*}$ ) is upper semicontinuous. Proposition 2.4 shows that Theorem 2.1 can be applied for $X:=(\operatorname{int} D(T),\|\cdot\|), F:=\left(E^{*},\|\cdot\|\right)$ and $G:=$ $E \subset E^{* *}$. We get in this way a dense $G_{i}$-subset $A$ of the strongly countably complete space $X=(\operatorname{int} D(T),\|\cdot\|)$ at each point $x_{0}$ of which the following is true:

(cp) $\left\{\begin{array}{l}\text { for every } \varepsilon>0 \text { there exists such a } \delta>0 \text { that }\left\|x^{\prime}-x_{0}\right\|<\delta \\ \text { and }\left\|x^{\prime \prime}-x_{0}\right\|<\delta \text { imply inf }\left\{\left\|e^{\prime *}-e^{\prime * *}\right\|: e^{\prime *} \in T x^{\prime}, e^{\prime \prime *} \in\right. \\ \left.T x^{\prime \prime}\right\} \leqq \varepsilon .\end{array}\right.$

How one can use this condition $(c p)$ to prove that $T$ is single- 
valued and upper semicontinuous at every point of $A$, is shown, in fact, in Lemma 2.9 (where instead of the norm $h(\cdot)$ in $E^{*}$ we take now the usual dual norm $\|\cdot\|$ of $E^{*}$ ). This completes the proof.

We give now a direct proof (without even mentioning the notion of dentability or the Radon-Nikodym property) of the following known fact (see Stegall [17]).

COROLlary 2.17. If every separable subspace of the Banach space $E$ has a separable dual space, then every continuous convex function $f: E \rightarrow R$ is Frechet differentiable at the points of some dense $G_{i}$-subset $A$ of $(E,\|\cdot\|)$.

Proof. As in the proof of Corollary 2.11 we consider the subgradient mapping $\partial: E \rightarrow E^{*}$ of the convex function $f: E \rightarrow R$. This subgradient is a monotone mapping and, by the previous result, there exists a dense $G_{i}$-subset $A$ of $(E,\|\cdot\|)$ at each point of which $\partial:(E,\|\cdot\|) \rightarrow\left(E^{*},\|\cdot\|\right)$ is single-valued and upper semicontinuous. That $f$ is Frechet differentiable at every $x_{0} \in A$ is proved, in fact, in Lemma 2.12 (where the norm $h(\cdot)$ of $E^{*}$ must be again replaced by the usual norm $\|\cdot\|$ of $\left.E^{*}\right)$.

3. Metric projections. We would like to describe here one more situation where the "continuity property" ( $c p)$ provides new information.

Let $M$ be such a subset of the Banach space $E$ that the corresponding metric projection $P: E \rightarrow M$ acting according to the rule $x \mapsto P(x):=\{y \in M:\|x-y\|=\inf \{\|x-z\|: z \in M\}\}$ has weak-compact images and is upper semicontinuous as a mapping from $(E,\|\cdot\|)$ into ( $M$, weak) (for instance, this is the case when the intersection of $M$ with any closed ball is a weakly compact set in $E$; the latter is fulfilled, in its turn, when $E$ is a reflexive Banach space and $M$ is weakly closed).

Define the mapping $T: E \rightarrow E$ by the formula $T x:=$ closed convex hull of the set $P(x)$. It is clear that $T:(E,\|\cdot\|) \rightarrow(E$, weak) is an upper semicontinuous mapping with weakly compact and convex images. Proposition 2.3 shows that Theorem 2.1 can be applied with $X$ and $F$ identical with $(E,\|\cdot\|)$ and $G=E^{*}$. As a result we get a dense $G_{o}$-subset $A$ of $(E,\|\cdot\|)$ such that, for every point $x_{0}$ of $A$, the following is true:

(cp) $\left\{\begin{array}{l}\text { for every } \varepsilon>0 \text { there exists such a } \delta>0 \text { that } \\ \left\|x^{\prime}-x_{0}\right\|<\delta \text { and }\left\|x^{\prime \prime}-x_{0}\right\|<\delta \text { imply inf }\left\{\left\|y^{\prime}-y^{\prime \prime}\right\|:\right. \\ \left.y^{\prime} \in T x^{\prime}, y^{\prime \prime} \in T x^{\prime \prime}\right\} \leqq \varepsilon .\end{array}\right.$

The key point now is the following 
LEMMA 3.1. If the Banach space $E$ is strictly convex (i.e., the equality $\|x+y\|=\|x\|+\|y\|$ implies $y=t x$ for some real number $t$ ) and the point $x_{0}$ belongs to the above defined set $A$, then the metric projection $P: E \rightarrow M$ is single-valued and norm-to-norm upper semicontinuous at $x_{0}$.

Proof. Let $\varepsilon>0$ and $\delta>0$ be as in the last $(c p)$ and $V:=$ $\left\{x \in E:\left\|x-x_{0}\right\|<\delta\right\}$. We prove now that the diameter of the set $P(V)=U\{P x: x \in V\}$ in $(E,\|\cdot\|)$ is smaller than $\varepsilon$. This is enough to conclude that $P: E \rightarrow M$ is single-valued and norm-to-norm upper semicontinuous at $x_{0}$. Take $x_{1}, x_{2} \in V$ and $y_{i} \in P\left(x_{i}\right), i=1,2$. When the real number $t>0$ is sufficiently small, the points $x^{\prime}=$ $t y_{1}+(1-t) x_{1}, x^{\prime \prime}=t y_{2}+(1-t) x_{2}$ will still belong to the open set $V$. By the strict convexity of the space $E, P\left(x^{\prime}\right)=y_{1}$ and $P\left(x^{\prime \prime}\right)=y_{2}$. Therefore for $T x^{\prime}$ and $T x^{\prime \prime}$, we have $T x^{\prime}=y_{1}$ and $T x^{\prime \prime}=y_{2}$. It follows now from $(c p)$ that $\left\|y_{1}-y_{2}\right\| \leqq \varepsilon$ and the diameter of $P(V)$ will also be smaller than $\varepsilon$. Thus we have proved:

THEOREM 3.2. Let $E$ be a strictly convex Banach space and suppose that the metric projection $P: E \rightarrow M$ has weak-compact images and is upper semicontinuous as a mapping from $(E,\|\cdot\|)$ into $\left(M\right.$, weak). Then there exists a dense $G_{\dot{o}}$-subset $A$ of $(E,\|\cdot\|)$ scuh that, at every point $x \in A$,

(i) $P: E \rightarrow M$ is single-valued.

(ii) $P:(E,\|\cdot\|) \rightarrow(M,\|\cdot\|)$ is upper semicontinuous.

It should be noted that (i) was already proved in another way in Kenderov [11]. The new information (due to $(c p)$ ) is contained in (ii) of the last theorem.

\section{REFERENCES}

1. A. Alexiewicz et W. Orlicz, Sur la continuité et la classification de Baire des fonctions abstraites, Fund, Math., 35 (1948), 105-126.

2. E. Asplund, Fréchet differentiability of convex functions, Acta Math., 121 (1968), $31-47$.

3. E. Asplund and R. T. Rockafellar, Gradients of convex functions, Trans. Amer. Math. Soc., 139 (1969), 433-467.

4. J. M. Borwein, Weak Local Supportability, Generic Gâteaux Differentiability and Applications to Approximation in a Weakly Compactly Generated Spaces, September 1977 (preprint).

5. J. Bourgain, Strongly exposed points in weakly compact, convex sets in Banach spaces, Proc. Amer. Math. Soc., 58 (1976), 197-200.

6. F. E. Browder, Nonlinear maximal monotone operators in Banach spaces, Math. Ann., 175 (1968), 89-113.

7. M. K. Fort, Category theorems, Fund. Math., 42 (1955), 276-288.

8. Z. Frolik, Generalizations of the $G_{\delta}$-property of complete metric spaces, Czech. 
Math. J., 10 (1960), 359-379.

9. P. Kenderov, The set-valued monotone mappings are almost everywhere singlevalued, Compt. Rendus de l'Académie bulgare des Sciences, 27 (1974), 1173-1175.

10. - Multivalued monotone mappings are almost everywhere single-valued, Studia Math., 56 (1976), 199-203.

11. - Uniqueness on a residual part of best approximations in Banach spaces. Pliska (Studia Math. bulgarica) v. 1 (1977), 122-127.

12. Monotone operators in Asplund spaces, Compt. Rendus de l'Académie bulgare des Sciences, 30 (1977), 963-964.

13. I. Namioka, Separate continuity and joint continuity, Pacific J. Math., 51 (1974), $515-531$.

14. I. Namioka and R. R. Phelps, Banach spaces which are Asplund spaces, Duke Math. J., 42 (1975), 735-750.

15. R. T. Rockafellar, Local boundedness of nonlinear monotone operators, Michigan Math. J., 16 (1969), 397-407.

16. C. Stegall, The Radon-Nikodym property in conjugate Banach spaces, Trans. Amer. Math. Soc., 206 (1975), 213-233.

17. - The duality between Asplund spaces and spaces with the Radon-Nikodym property, Israel J. Math., 29 (1978), 408-412.

18. J.-P. Troallic, Fonctions à valeurs dans des espaces fonctionnels généraux: théorémes de R. Ellis et de I. Namioka, C. R. Acad. Sc. Paris, 287 (1978), Série A63-66.

19. S. Trojansky, On locally convex and differentiable norms in certain nonseparable Banach spaces, Studia Math., 37 (1971), 173-180.

20. J. J. Uhl, A note on the Radon-Nikodym theorem for Banach spaces, Rev. Roum. Math. Pure et Appl., 17 (1972), 113-115.

Received March 7, 1979 and in revised form July 24, 1979. This paper was written while the author was at the University of Frankfurt am Main as a research fellow of the Alexander von Humboldt Foundation.

Bulgarian Academy of Sciences

1090 Sofia, P.O. Box 373

BUlgaria

AND

Fachbereich Mathematik,

JoHanN Wolfgang Goethe-Universität

ROBERT-MaYer-STRAßE 6-10,

6000 Frankfurt am Main, West Germany 


\section{PACIFIC JOURNAL OF MATHEMATICS}

\section{EDITORS}

DONALD BABBITT (Managing Editor)

University of Galifornia

Los Angeles, California 90024

Hugo RossI

University of Utah

Salt Lake City, UT 84112

C. C. MoOre AND ANDrew OGG

University of California

Berkeley, CA 94720

\section{J. DugundjI}

Department of Mathematics University of Southern California

Los Angeles, California 90007

R. Finn and J. Milgram

Stanford University

Stanford, California 94305

ASSOCIATE EDITORS

E. F. Beckenbachi

B. H. NeumanN

F. WOLF

K. YOSHIDA

\section{SUPPORTING INSTITUTIONS}

UNIVERSITY OF BRITISH COLUMBIA

CALIFORNIA INSTITUTE OF TECHNOLOGY

UNIVERSITY OF CALIFORNIA

MONTANA STATE UNIVERSITY

UNIVERSITY OF NEVADA, RENO

NEW MEXICO STATE UNIVERSITY

OREGON STATE UNIVERSITY

UNIVERSITY OF OREGON
UNIVERSITY OF SOUTHERN CALIFONIA

STANFORD UNIVERSITY

UNIVERSITY OF HAWAII

UNIVERSITY OF TOKYO

UNIVERSITY OF UTAH

WASHINGTON STATE UNIVERSITY

UNIVERSITY OF WASHINGTON

Printed in Japan by International Academic Printing Co., Ltd., Tokyo, Japan 


\section{Pacific Journal of Mathematics \\ Vol. 89 , No. 1 \\ May, 1980}

David Bressoud, A note on gap-frequency partitions ................ 1

John David Brillhart, A double inversion formula ................. 7

Frank Richard Deutsch, Günther Nürnberger and Ivan Singer, Weak

Chebyshev subspaces and alternation .......................

Edward Richard Fadell, The relationship between Ljusternik-Schnirelman

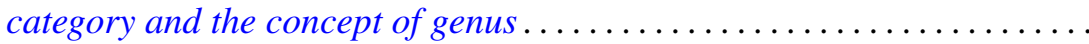

Harriet Jane Fell, On the zeros of convex combinations of polynomials.......

John Albert Fridy, An addendum to: "Tauberian theorems via block

dominated matrices" ..................................

Andrzej Granas, Ronald Bernard Guenther and John Walter Lee, Applications of topological transversality to differential equations. I. Some nonlinear

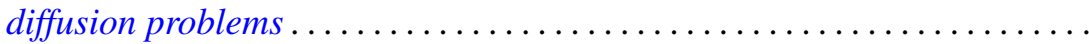

David E. Handelman and G. Renault, Actions of finite groups on self-injective

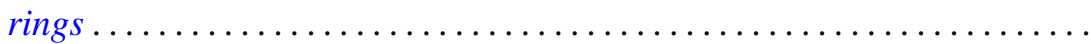

Michael Frank Hutchinson, Local $\Lambda$ sets for profinite groups .............

Arnold Samuel Kas, On the handlebody decomposition associated to a

Lefschetz fibration...

Hans Keller, On the lattice of all closed subspaces of a Hermitian space.....

P. S. Kenderov, Dense strong continuity of pointwise continuous

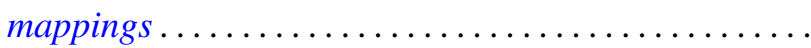

Robert Edward Kennedy, Krull rings.................

Jean Ann Larson, Richard Joseph Laver and George Frank McNulty,

Square-free and cube-free colorings of the ordinals ...

Viktor Losert and Harald Rindler, Cyclic vectors for $L^{p}(G)$

John Rowlay Martin and Edward D. Tymchatyn, Fixed point sets of

1-dimensional Peano continua...

Augusto Nobile, On equisingular families of isolated singularities ...

Kenneth Joseph Prevot, Imbedding smooth involutions in trivial bundles ...

Thomas Munro Price, Spanning surfaces for projective planes in four space.

Dave Riffelmacher, Sweedler's two-cocycles and Hochschild cohomology....

Niels Schwartz, Archimedean lattice-ordered fields that are algebraic over

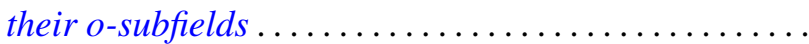

Chao-Liang Shen, A note on the automorphism groups of simple dimension groups.

Kenneth Barry Stolarsky, Mapping properties, growth, and uniqueness of

Vieta (infinite cosine) products ...

Warren James Wong, Maps on simple algebras preserving zero products. I.

The associative case ............................. 